\title{
Single-center outcomes for percutaneous pedicle screw fixation in metastatic spinal lesions: can spontaneous facet fusion occur?
}

\author{
Ahmed Meleis, MD, ${ }^{1}$ M. Benjamin Larkin, MD, PharmD, ${ }^{2}$ Dhiego Chaves de Almeida Bastos, MD, ${ }^{1}$ \\ Matthew T. Muir, BS, ${ }^{3}$ Ganesh Rao, MD, ${ }^{1}$ Laurence D. Rhines, MD, ${ }^{1}$ Charles E. Cowles, MD, ${ }^{1}$ and \\ Claudio E. Tatsui, MD1
}

${ }^{1}$ Department of Neurosurgery, MD Anderson Cancer Center; and ${ }^{2}$ Department of Neurosurgery and ${ }^{3}$ School of Medicine, Baylor College of Medicine, Houston, Texas

\begin{abstract}
OBJECTIVE Survival of cancer patients continues to improve with systemic treatment advancements, leading to an increase in cancer-related complications such as pathological spinal fractures. In this study, the authors aimed to evaluate the outcome of percutaneous stabilization with cement augmentation of the pedicle screws in the management of patients with metastatic cancer to the spine.

METHODS The authors reviewed a retrospective case series of 74 patients with symptomatic pathological spine fractures treated with cement-augmented pedicle screws implanted with a percutaneous technique. The mean imaging follow-up was 11.3 months. Data on demographics, clinical outcomes, and complications were collected. Cement extravasation, spinal hardware integrity, and fusion rates were assessed on CT scans.

RESULTS Among 50 patients with follow-up imaging, 23 patients (46\%) showed facet joint fusion. The length of segmental stabilization was not a significant predictor of the occurrence of fusion. Pre- or postoperative radiation therapy, postoperative chemotherapy, and the location of spinal lesions did not have a statistically significant effect on the occurrence of fusion. Patients older than 60 years of age were more likely to have fusion across facet joints compared with younger patients. There was a significant difference in the mean visual analog scale pain score, with 6.28 preoperatively and 3.41 postoperatively, regardless of fusion status $(p<0.001)$. Cement extravasation was seen in $51 \%$ of the cohort, but in all instances, patients remained asymptomatic. Most importantly, the incidence of hardware failure was low (4\%).

CONCLUSIONS Percutaneous fixation with cement-augmented pedicle screws in patients with pathological spine fractures provides an improvement in mechanical back pain, with a low incidence of failure, and in some patients, spontaneous facet fusion was observed. Further research is necessary with regard to both short-term benefits and long-term outcomes.
\end{abstract}

https://thejns.org/doi/abs/10.3171/2021.1.FOCUS20671

KEYWORDS cancer pain; cement augmentation; pathological fracture; percutaneous fixation; spinal fixation

$\mathrm{S}$ URVIVAL in many patients with cancer continues to improve as significant advancements in genomics, diagnostics, and local and systemic treatments become available; thus, the incidence of malignancy-related complications like vertebral body fractures is expected to increase. ${ }^{1}$ The Spine Oncology Study Group (SOSG) defines spinal instability as "loss of spinal integrity as a result of a neoplastic process that is associated with movement-related pain, symptomatic or progressive deformity, and/or neural compromise under physiologic loads." ${ }^{2}$ The
SOSG also developed a novel classification system to assess the stability of the spine in patients with metastatic spinal disease, known as the Spine Instability Neoplastic Score (SINS). This system helped improve communication among treating physicians and standardized the assessment of spinal instability. ${ }^{3}$ Cancer-related spinal fractures are unlikely to heal with nonsurgical management given the ongoing destruction of bone by the underlying disease or the result of adjuvant treatments, i.e., radiation and chemotherapy, which negatively impact bone repair, remodel-

ABBREVIATIONS DVT = deep vein thrombosis; IVC = inferior vena cava; LOS = length of hospital stay; PE = pulmonary embolism; PMMA = polymethylmethacrylate; VAS = visual analog scale. 
ing, and fusion capacity. Comorbidities such as malnourishment, osteoporosis, need for anticoagulation, immunosuppression, diabetes, and chronic use of steroids are often present in these patients, which increases the risk of complications in open procedures for spinal stabilization. The utilization of minimally invasive spinal stabilization techniques in selected cases can be advantageous as less trauma to soft tissue often results in lower wound-related complications, less postoperative pain, and faster implementation of postoperative radiation and systemic therapy. Moussazadeh et al. ${ }^{4}$ described the utilization of cementaugmented percutaneous pedicle screws allowing patients to safely undergo radiation therapy within 4 weeks of surgery, with some of their patients starting adjuvant therapy within a week from the procedure. Furthermore, their study found the proportion of patients with severe pain who underwent percutaneous stabilization decreased from $86 \%$ preoperatively to $0 \%$ postoperatively. Unfortunately, there was only a short follow-up, and the radiographic evaluation of hardware durability was limited.

The objective of the present study is to report outcomes of percutaneous stabilization using cement augmentation of pedicle screws in the management of spinal metastasis and to provide an analysis of the radiographic evaluation of hardware integrity and fusion.

\section{Methods \\ Patient Selection}

This study is a single-center, descriptive, retrospective chart and imaging review for cases performed between April 2015 and August 2019. We included patients with neoplastic spinal instability who underwent percutaneous instrumented spinal stabilization with cement augmentation of fenestrated pedicle screws with clinical, radiological follow-up for at least 6 weeks postoperatively. Patients were more likely to undergo cement augmentation, as opposed to kyphoplasty/vertebroplasty, if there was a > $70 \%$ vertebral collapse associated with significant osteolysis and disruption of the posterior wall of the vertebral body. Patients who underwent open posterior stabilization, those whose constructs were not augmented with polymethylmethacrylate (PMMA), those who underwent direct PMMA augmentation without the use of fenestrated pedicle screws, and those with follow-up less than 6 weeks were excluded. The IRB committee approved the study. All data were kept in accordance with HIPAA regulations.

\section{Surgical Technique}

The procedure of percutaneous stabilization with cement augmentation has been previously described in the literature. ${ }^{1,4,5}$ The authors used Viper fenestrated screws (DePuy Synthes) and VertaPlex bone cement (Stryker). All pedicle screws were inserted with a percutaneous technique. PMMA was injected bilaterally at each level through the fenestrated screws under fluoroscopic guidance. In the thoracic spine from $\mathrm{T} 1$ to $\mathrm{T} 6$, at least $2 \mathrm{ml}$ was injected per level; from T7 to T12, $3 \mathrm{ml}$ was typically used. In the lumbar spine, at least $4 \mathrm{ml}$ was injected. The goal was to visualize a cloud of cement around the pedicle screw in both anteroposterior and lateral fluoroscopic im- ages. If any extravasation was seen prior to reaching the expected volume, the injection was immediately ceased. Lastly, subfascial interconnecting rods were placed and secured.

Patients were evaluated within 1 month of surgery to assess pain scale and postoperative checks. Individuals were followed up at 3 and 6 months postoperatively and annually afterward. Data collection included demographics, location of spine tumor, pre- and postoperative visual analog scale (VAS) pain score, as well as preoperative and postoperative radiation therapy. Treatment variables included the number of spinal segments instrumented, intraoperative adverse events, and the length of hospital stay (LOS). Postoperative data included the need for additional spinal surgery, PMMA-related complications (pulmonary embolism [PE] or deep vein thrombosis [DVT]), and medical complications within 30 days postoperatively, including pneumonia, wound healing problems, PE/DVT, and urinary tract infections.

All patients underwent postoperative CT scanning to confirm appropriate hardware placement and assessment of cement extravasation that may have occurred intraoperatively and not identified on fluoroscopy images. All individuals had postoperative CT scanning at regular intervals for cancer restaging, and these scans were used to evaluate the integrity of the spinal hardware and determine if the facet joints had fused across the instrumented segments. The occurrence of hardware failure was defined as 1) lucency around the pedicle screws, 2) rod or screw breakage, 3) loosening of set screws, or 4) subsidence of pedicle screws. Fusion was defined as the complete union with cartilage reabsorption across the facet joints at the stabilized segments. The time to fusion was defined by the interval between the initial postoperative CT scan to the first subsequent scan demonstrating fusion as defined above. The number of segments instrumented was divided into two groups: a short-segment group (1 level above and 1 level below the vertebral body fracture), and a long-segment group (where the instrumented levels were 2 or more levels above and below the diseased segment) (Fig. 1). For lesions located between $\mathrm{T} 1$ and $\mathrm{T} 11$, there is a preference for short-segment constructs. In patients with lesions involving 2 or more adjacent levels, the thoracolumbar junction, or the lumbar spine, longer constructs are more likely to be utilized due to higher axial loads.

\section{Statistical Analysis}

SPSS Statistics version 22.0 (IBM Corp.) was used for statistical analysis. Descriptive statistics, including medians, means, standard deviations, and frequencies, were used to define the cohort. Categorical variables for the groups were compared with a Pearson chi-square test. The Student t-test was used for continuous variables. Logistic regression was performed to evaluate whether there were variables affecting fusion rates among patients. A p value $<0.05$ was considered statistically significant.

\section{Results}

\section{Demographics and Clinical Results}

We identified 74 patients who met the study criteria. 

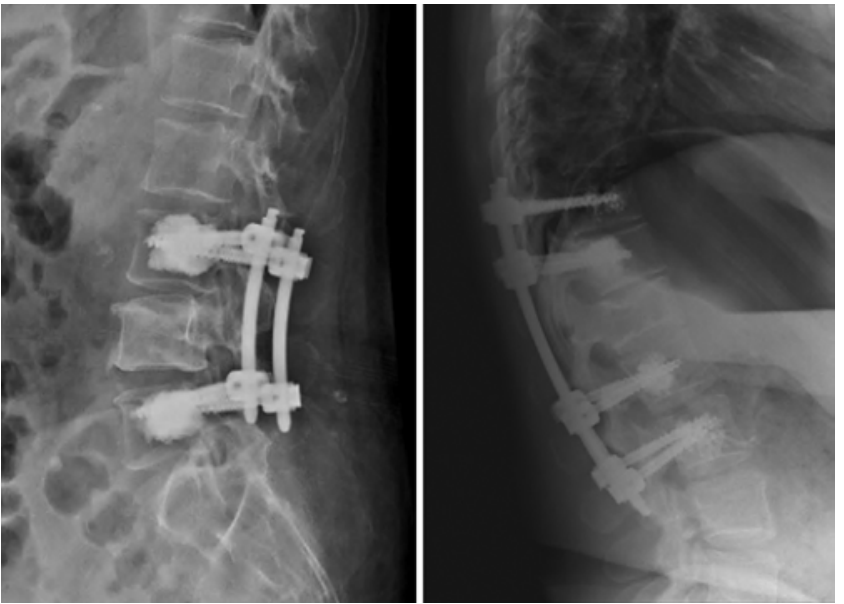

FIG. 1. Lateral radiographs. Left: Short-segment fusion in which the instrumented levels are no more than 1 level above and 1 level below the fractured segment. Right: Long-segment fusion in which the instrumented levels are more than 1 level above and 1 level below the fractured segment.

These patients underwent percutaneous stabilization with cement-augmented pedicle screws. There were 35 females and 39 males $(p=0.809)$. The most commonly treated area was thoracic spine $(n=20)$, followed by the thoracolumbar junction $(\mathrm{n}=16)$, lumbar $(\mathrm{n}=9)$, and lumbosacral $(n=4)$ regions. Forty-three patients $(58 \%)$ had radiation therapy preoperatively, while 33 patients $(44.6 \%)$ had radiation therapy postoperatively. Additionally, 41 (55\%) patients had chemotherapy postoperatively. The difference between preoperative and postoperative VAS scores was statistically significant $(\mathrm{p}<0.001)$. The mean preoperative VAS score was 6.28 (median 7), while the mean postoperative VAS score was 3.41 (median 3.0). The median time to first follow-up, during which the postoperative pain score was obtained, was 24.2 days (SD 12.2 days). The median length of clinical follow-up was 5 months.

\section{Complications}

Thirty-eight patients (51\%) had asymptomatic radiographic extravasation of cement outside the vertebral body into the prevertebral veins adjacent to the vertebral body or the surrounding soft tissue. One patient $(1.3 \%)$ had cement extravasation into the inferior vena cava (IVC) tracking into the intrahepatic segment. This patient was treated with full-dose anticoagulation for 6 months and remained asymptomatic. One patient (1.3\%) had a surgical site muscular hematoma. Ten patients $(13.5 \%)$ had postoperative medical complications: 3 had DVTs, and 2 had a PE associated with a DVT; 4 patients had postoperative pneumonia, while 1 patient had a pleural effusion secondary to the underlying primary malignancy. There were no postoperative mortalities secondary to the operative intervention. The mean LOS was 6.9 days, with a median of 5 days. While the overall surgical complication rate was 54\% (40 patients), there was no significant difference in the LOS (p $=0.541)$ or postoperative pain $(\mathrm{p}=0.131)$ among patients with cement extravasation, and all instances of cement extravasation were asymptomatic.
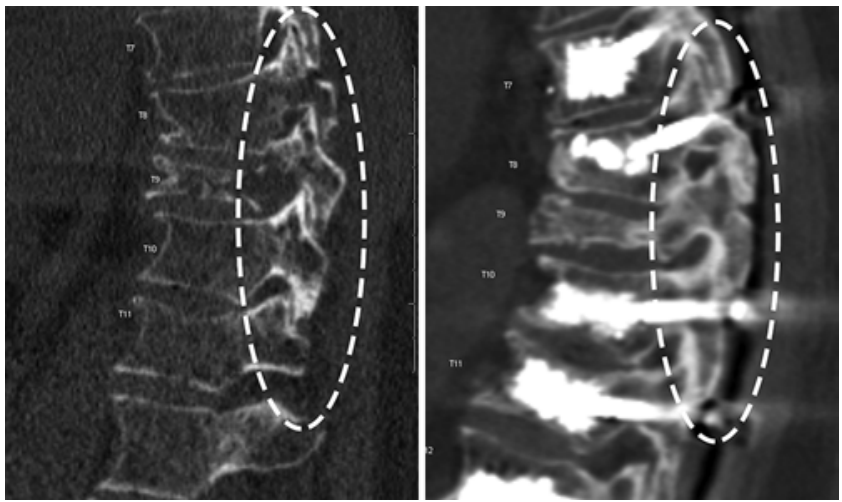

FIG. 2. Sagittal CT scans demonstrating the presence or absence of posterior fusion after percutaneous stabilization. Left: Scan obtained preoperatively showing that the joints are not ankylosed. Right: Scan obtained 12 months postoperatively demonstrating spontaneous ankyloses of the posterior joints between the segments (circle).

\section{Durability of Stabilization}

Hardware was intact in $96 \%$ of patients at the median follow-up of 5 months. The overall incidence of hardware failure was $4 \%$. Three patients had hardware failure: one was involved in a car accident that caused a rod fracture requiring operative repair, another was found to have a broken screw 18 months following the initial operation, and the last patient had lucency surrounding one of the screws 3 months postoperatively. The latter two did not require surgical intervention as the patients were asymptomatic.

\section{Spontaneous Facet Fusion}

Follow-up CT scans were available in 50 patients with a median interval of 11.3 months. The remaining 24 patients were lost to follow-up. Of these 50 patients, 27 (54\%) demonstrated nonfusion.

Twenty-three patients (46\%) showed evidence of spontaneous facet fusion; of those, 11 had short-segment constructs (47.8\%) and 12 had long-segment constructs (52.2\%) (Fig. 2). The length of segmental stabilization was not a significant predictor of the occurrence of fusion $(\mathrm{p}=$ 0.283 ). Whether radiation therapy was completed pre- or postoperatively did not influence the occurrence of bony fusion. Furthermore, sex, postoperative chemotherapy, and fracture location (thoracic, lumbar, thoracolumbar, or lumbosacral) were not significant predictors of fusion rates.

Additionally, there was no significant difference in the number of complications between patients who underwent fusion and those who did not. The only factor influencing facet fusion was patient age; individuals older than 60 years had significantly more facet fusion than those younger than 60 years $(p=0.019)$. There was no significant difference in hardware failure between the fusion and nonfusion groups $(\mathrm{p}=0.459)$. Univariate analysis of variables influencing fusion rates is depicted in Table 1.

\section{Discussion}

Pathological fractures from metastatic spinal disease resulting in spinal instability often require stabilization as 
TABLE 1. Patient variables for fusion versus nonfusion

\begin{tabular}{|c|c|c|c|}
\hline Variable & $\begin{array}{c}\text { Fusion } \\
\text { Group }(n=23)\end{array}$ & $\begin{array}{c}\text { Nonfusion } \\
\text { Group }(n=27)\end{array}$ & $p$ Value \\
\hline \multicolumn{4}{|l|}{ Sex } \\
\hline Male & $9(39.1)$ & $14(51.9)$ & \\
\hline Female & $14(60.9)$ & $13(48.1)$ & 0.809 \\
\hline Median age, yrs (SD) & $64(8.0)$ & $56(11.52)$ & 0.032 \\
\hline Age $>60$ yrs & $17(73.9)$ & $11(40.7)$ & \\
\hline Age $<60$ yrs & $6(26.1)$ & $16(59.3)$ & 0.019 \\
\hline \multicolumn{4}{|l|}{ Segments } \\
\hline Short & $11(47.8)$ & $17(63)$ & \\
\hline Long & $12(52.2)$ & $10(37)$ & 0.283 \\
\hline \multicolumn{4}{|l|}{ Preop radiotherapy } \\
\hline No & $9(39.1)$ & $11(40.7)$ & \\
\hline Yes & $14(60.9)$ & $16(59.3)$ & 0.908 \\
\hline \multicolumn{4}{|l|}{ Postop radiotherapy } \\
\hline No & $13(56.5)$ & $13(48.1)$ & \\
\hline Yes & $10(43.5)$ & $14(51.9)$ & 0.555 \\
\hline \multicolumn{4}{|l|}{ Postop chemotherapy } \\
\hline No & $10(43.5)$ & $16(59.3)$ & \\
\hline Yes & $13(56.5)$ & $11(40.7)$ & 0.266 \\
\hline \multicolumn{4}{|l|}{$\begin{array}{l}\text { Postop complications } \\
\text { (cement) }\end{array}$} \\
\hline No & $10(43.5)$ & $13(48.1)$ & \\
\hline Yes & $13(56.5)$ & $14(51.9)$ & 0.741 \\
\hline \multicolumn{4}{|l|}{ Postop complications } \\
\hline No & $21(91.3)$ & $22(81.5)$ & \\
\hline Yes & $2(8.7)$ & $5(18.5)$ & 0.318 \\
\hline \multicolumn{4}{|l|}{ Hardware failure } \\
\hline No & $21(91.3)$ & $26(96.3)$ & \\
\hline Yes & $2(8.7)$ & $1(3.7)$ & 0.459 \\
\hline \multicolumn{4}{|l|}{ Levels } \\
\hline Thoracic & $8(34.8)$ & $12(44.4)$ & \\
\hline Lumbar & $3(13)$ & $6(22.2)$ & \\
\hline Thoracolumbar & $8(34.8)$ & $8(29.6)$ & \\
\hline Lumbosacral & $3(13)$ & $1(3.7)$ & 0.557 \\
\hline Median LOS, days & 5 & 5 & 0.296 \\
\hline $\begin{array}{l}\text { Mean VAS pain score } \\
\text { preop (SD) }\end{array}$ & $4.87(2.3)$ & $6.3(2.35)$ & 0.762 \\
\hline $\begin{array}{l}\text { Mean VAS pain score } \\
\text { postop (SD) }\end{array}$ & $3.13(2.16)$ & $3.00(2.4)$ & 0.420 \\
\hline
\end{tabular}

Values represent the number of patients (\%) unless stated otherwise.

these patients have a low likelihood of bone healing with nonsurgical methods. ${ }^{6}$ This is likely due to the effects of systemic chemotherapy, prior spinal radiation therapy, and local tumor burden. ${ }^{6}$ Surgical intervention in the metastatic cancer population is palliative, and these patients should be considered for less-invasive procedures that limit the interruption of systemic therapy and allow for the delivery of early adjuvant radiation. ${ }^{4}$

Minimally invasive surgical techniques have been used in spine trauma, deformity, and degenerative disease, with these same principles now being utilized in patients with cancer-associated mechanical pain., ${ }^{1,47-16}$ Vertebroplasty and kyphoplasty have historically been used to relieve pathological fractures. In our experience, there is a higher risk of cement extravasation and suboptimal vertebral augmentation when there is a vertebral collapse of more than $70 \%$, associated with significant osteolysis and disruption of the posterior vertebral body wall. Additionally, in patients with significant disruption of the vertebral body walls, these methods are often not adequate for fractures that further extend into the pedicles, facet joints, and spinous process, especially at junctional levels. In contrast, pedicle screw stabilization provides further reinforcement to both the anterior and posterior columns. ${ }^{17-22}$ In such cases, percutaneous stabilization with pedicle screws in the levels adjacent to the pathological fracture allows for quick restoration of the axial and rotational load capacities of the spine. Percutaneous screw placement has some shortcomings, especially in this patient population, including the inability for bone graft placement or sufficient decortication to further encourage posterolateral fusion, as occurs with more-invasive open techniques. Therefore, a solid arthrodesis is not expected, and this technique is more susceptible to failure over time as the bone remodels at the bone-screw interface, resulting in micromotion around the screw, hardware loosening, and eventually weakening the fixation.

Biomechanical studies have demonstrated that cement augmentation of the pedicle screws significantly increases the screw pullout strength and can translate to a more durable stabilization in the absence of a solid arthrodesis. ${ }^{23,24}$ Barzilai et al. showed that cement augmentation of pedicle screws is a safe and effective method to enhance the durability of spinal constructs in the cancer population. ${ }^{1}$ In their series of 53 patients, there were no intraoperative adverse events requiring modification.

Our clinical experience has been very positive with cement-augmented screws (open or percutaneous). We believe that they provide better load sharing due to the wider contact area provided by the bone/screw/cement interface, thereby reducing the likelihood of a "halo" effect around the screw, and consequently improving the durability of the reconstruction, especially in short-segment stabilizations. In the setting of neoplastic disease, a combination of cement augmentation and percutaneous instrumented stabilization represents a minimally invasive treatment option to restore spinal stability. Most importantly, this technique allows for a decreased time interval to radiation therapy and systemic therapy compared with conventional open posterolateral instrumented fusion. ${ }^{4}$

After percutaneous stabilization in spinal metastasis patients, complications have been shown to be relatively high (18\%) but remain comparatively less than several open retrospective and prospective studies following open surgical procedures $(15 \%-47 \%)$. $^{25,26}$ Versteeg et al. reported on 6 patients $(6 \%)$ who required reoperation; with 3 resulting from construct failure and 1 from medial placement of the pedicle screw..$^{25}$ Three patients had cement-augmented stabilization, with 1 patient who sustained an incomplete spinal cord injury (American Spinal Injury Association Impairment Scale grade C) due to cement extravasation, but who recovered fully following reoperation. ${ }^{25}$ 

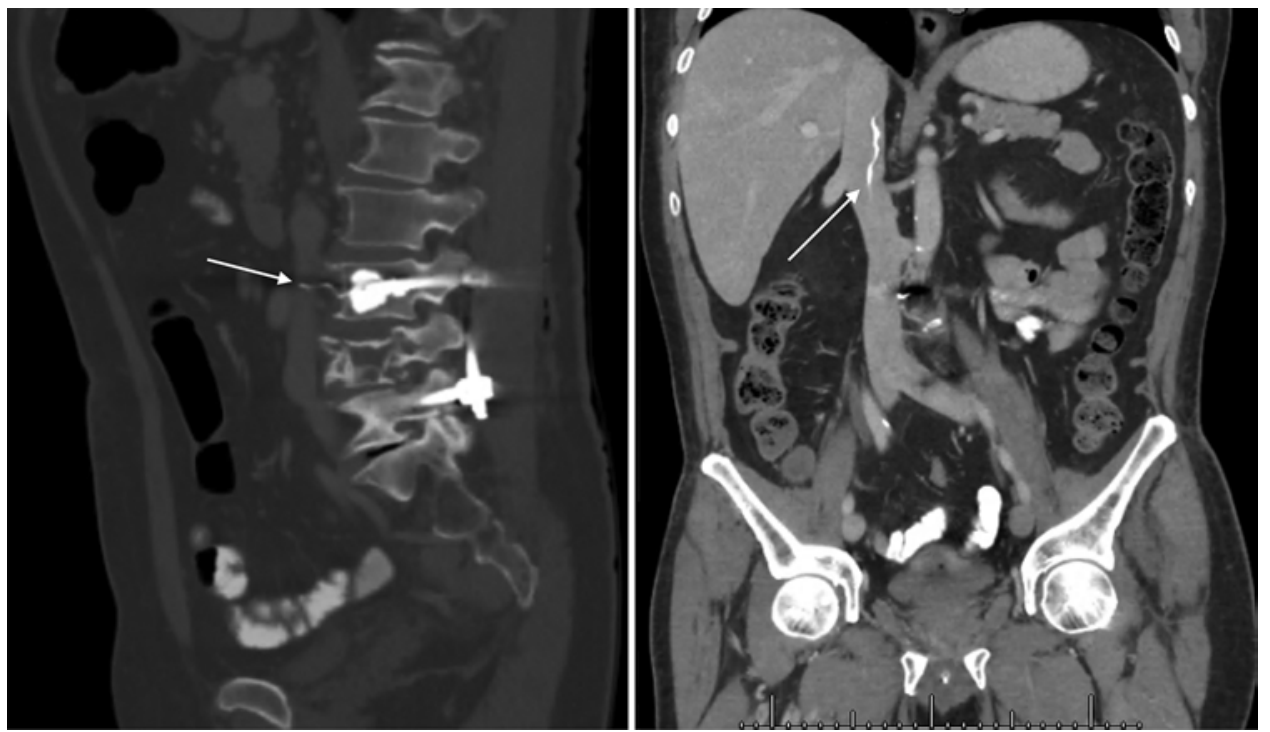

FIG. 3. Left: Sagittal CT scan demonstrating cement extravasation into the prevertebral veins (arrow). Right: Coronal CT scan showing the cement extravasation into the intrahepatic segment of the IVC (arrow) in 1 patient in our cohort. The patient was prescribed full-dose anticoagulation for 6 months.

In this cohort of 74 patients who underwent percutaneous stabilization with cement augmentation, no patients had extravasation of cement into the spinal canal. Thirtyeight patients had some extravasation of cement outside of the vertebral body into the prevertebral veins adjacent to the vertebral body or the surrounding soft tissue. This had no impact on patient outcomes, as there was no significant difference in LOS or pain relief compared with patients without cement extravasation.

One of our patients had evidence of cement embolism into the IVC, which extended into the intrahepatic segment of the IVC (Fig. 3). Although standard treatment of a PE requires 3 to 6 months of systemic anticoagulation, some studies have shown that PMMA is not thrombogenic; thus, the need for anticoagulation following asymptomatic cement PE remains debatable..$^{24,27-29}$ This patient remained asymptomatic but was started on 6 months of therapeutic anticoagulation.

The incidence of hardware failure was low, and only 1 of the 50 patients with long-term follow-up required hardware revision. This revision was needed because the patient was in a car accident 3 months after surgery, which resulted in the fracture of a rod. The patient remained asymptomatic and without any neurological deficit. One patient was found to have an asymptomatic screw breakage at 18 months postsurgery, and 1 patient developed lucency around one of the pedicle screws 3 months postoperatively. Both patients remained asymptomatic throughout clinical and radiographic follow-up. We believe that the extremely low incidence of a halo effect around the pedicle screws was due to a decrease in micromotion at the screw/cement/bone junction, where the cement absorbs and distributes compression and extension forces to a larger area, decreasing stress in the surrounding bone and decreasing the remodeling around the pedicle screws.

Cancer patients who undergo spinal fusion immediately followed by local radiation therapy and chemotherapy are less likely to develop spinal arthrodesis. ${ }^{1,4}$ Imaging followup longer than 3 months was available in 50 patients, and this group was specifically analyzed for the occurrence of spontaneous facet fusion, which was observed in 23 patients (46\%). There was no statistical significance between the short- and long-segment stabilization procedures with respect to fusion, the number of complications, hardware failure, and postoperative radiation, which did not influence facet fusion development. Furthermore, sex, postoperative chemotherapy, or location of the pathological fracture (thoracic, lumbar, thoracolumbar, or lumbosacral) did not influence fusion rates. Interestingly, when broken down between two age groups (patients $>60$ years and patients $<60$ years), there was a statistically significant difference in fusion rates. Specifically, patients older than 60 years of age were more likely to have fusion across facet joints than younger patients. We postulate that older individuals may have a predisposition for spine ankylosis, as their spines tend to be stiffer due to spondylotic changes. The placement of the pedicle screw construct further immobilizes the joints accelerating the absorption of cartilage and fusion of the facet surfaces.

A significant improvement in the preoperative VAS score was observed regardless of the development of facet fusion (Fig. 4). The pain improvement was reported within the 1-month follow-up and remained lower than baseline during the entire follow-up period.

This study has several limitations, including its retrospective nature, the lack of a control group undergoing open spinal stabilization, or a group of patients undergoing placement of pedicle screws without cement augmentation. There was no control over the type and/or interval for the administration of radiation and systemic treatments, and patients presented with vastly different stages of disease. Additionally, there were no specific criteria for 


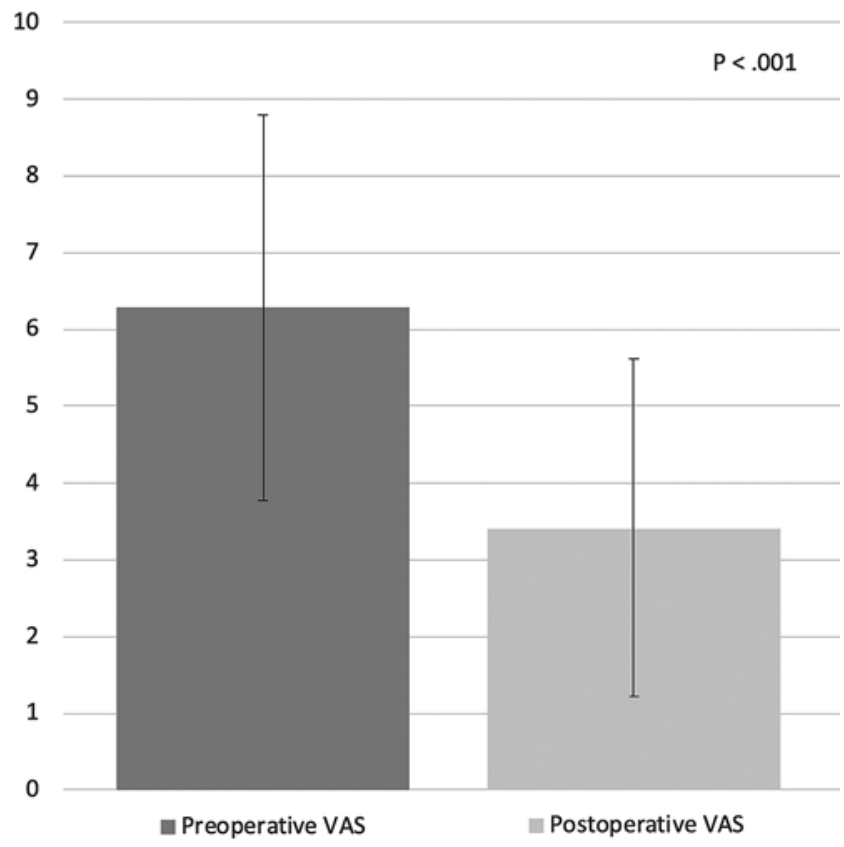

FIG. 4. Bar graph showing an improvement in the mean VAS pain score following cement-augmented percutaneous pedicle screw fixation for metastatic spinal lesions. Error bars represent the SD.

the selection of short versus long constructs. However, we believe that our cohort represents a "real world" population receiving care in a tertiary cancer center over a given time and that our results indicate that these individuals can benefit from percutaneous pedicle screw stabilization without the need for bone grafting.

\section{Conclusions}

Surgical intervention for mechanical instability secondary to spinal metastases is evolving, with safer and less-invasive techniques. Percutaneous pedicle screw fixation with cement augmentation reduces the radiographic incidence of screw loosening even when a solid arthrodesis is not achieved and offers a significant and durable decrease in mechanical back pain..$^{1,4}$ Our experience further supports these reports. Patients older than 60 years of age, who are more likely to have some degree of preoperative ankylosis, consequently had a significantly higher rate of spontaneous facet fusion. To our knowledge, spontaneous facet fusion has not been previously reported in this population and may prove to be relevant in long-term outcomes of cancer patients as survival continues to improve. More importantly, patients in this cohort experienced significant improvement in the postoperative pain score, and there was a low rate of complications following cement-augmented percutaneous fixation. Continued research regarding both short-term benefits and long-term outcomes in pain reduction, fusion, functional status, and overall quality-of-life measures, that improve patient outcomes and justify the increased cost, are needed when evaluating percutaneous fixation with cement augmentation in patients with spinal metastases.

\section{References}

1. Barzilai O, McLaughlin L, Lis E, et al. Utility of cement augmentation via percutaneous fenestrated pedicle screws for stabilization of cancer-related spinal instability. Oper Neurosurg (Hagerstown). 2019;16(5):593-599.

2. Fisher CG, Dipaola CP, Ryken TC, et al. A novel classification system for spinal instability in neoplastic disease an evidence-based approach and expert consensus from the Spine Oncology Study Group. Spine (Phila Pa 1976). 2010;35(22): E1221-E1229.

3. Fourney DR, Frangou EM, Ryken TC, et al. Spinal instability neoplastic score: an analysis of reliability and validity from the Spine Oncology Study Group. J Clin Oncol. 2011;29(22): 3072-3077.

4. Moussazadeh N, Rubin DG, McLaughlin L, et al. Shortsegment percutaneous pedicle screw fixation with cement augmentation for tumor-induced spinal instability. Spine J. 2015;15(7):1609-1617.

5. Vega RA, Traylor JI, Habib A, et al. Minimally invasive separation surgery for metastases in the vertebral column: a technical report. Oper Neurosurg (Hagerstown). 2020;18(6): 606-613.

6. Rades D, Fehlauer F, Schulte R, et al. Prognostic factors for local control and survival after radiotherapy of metastatic spinal cord compression. J Clin Oncol. 2006;24(21):3388-3393.

7. Oppenheimer JH, DeCastro I, McDonnell DE. Minimally invasive spine technology and minimally invasive spine surgery: a historical review. Neurosurg Focus. 2009;27(3):E9.

8. Holly LT, Schwender JD, Rouben DP, Foley KT. Minimally invasive transforaminal lumbar interbody fusion: indications, technique, and complications. Neurosurg Focus. 2006;20(3): E6.

9. Kim CW. Scientific basis of minimally invasive spine surgery: prevention of multifidus muscle injury during posterior lumbar surgery. Spine (Phila Pa 1976). 2010;35(26)(suppl): S281-S286.

10. Kim DY, Lee SH, Chung SK, Lee HY. Comparison of multifidus muscle atrophy and trunk extension muscle strength: percutaneous versus open pedicle screw fixation. Spine (Phila Pa 1976). 2005;30(1):123-129.

11. Kanter AS, Mummaneni PV. Minimally invasive spine surgery. Neurosurg Focus. 2008;25(2):E1.

12. Chou D, Lu DC. Mini-open transpedicular corpectomies with expandable cage reconstruction. Technical note. J Neurosurg Spine. 2011;14(1):71-77.

13. Assaker R. Minimal access spinal technologies: state-ofthe-art, indications, and techniques. Joint Bone Spine. 2004; 71(6):459-469.

14. Selznick LA, Shamji MF, Isaacs RE. Minimally invasive interbody fusion for revision lumbar surgery: technical feasibility and safety. J Spinal Disord Tech . 2009;22(3):207-213.

15. Kerr SM, Tannoury C, White AP, et al. The role of minimally invasive surgery in the lumbar spine. Oper Tech Orthop. 2007;17(3):183-189.

16. Hsieh PC, Koski TR, Sciubba DM, et al. Maximizing the potential of minimally invasive spine surgery in complex spinal disorders. Neurosurg Focus. 2008;25(2):E19.

17. Ni WF, Huang YX, Chi YL, et al. Percutaneous pedicle screw fixation for neurologic intact thoracolumbar burst fractures. J Spinal Disord Tech. 2010;23(8):530-537.

18. Chi JH, Gokaslan ZL. Vertebroplasty and kyphoplasty for spinal metastases. Curr Opin Support Palliat Care. 2008; 2(1):9-13.

19. Jha RM, Hirsch AE, Yoo AJ, et al. Palliation of compression fractures in cancer patients by vertebral augmentation: a retrospective analysis. J Neurointerv Surg. 2010;2(3):221-228.

20. Sun G, Li L, Jin P, et al. Percutaneous vertebroplasty for painful spinal metastasis with epidural encroachment. J Surg Oncol. 2014;110(2):123-128. 
21. Moussazadeh N, Laufer I, Werner T, et al. Sacroplasty for cancer-associated insufficiency fractures. Neurosurgery. 2015;76(4):446-450.

22. Bilsky MH, Lis E, Raizer J, et al. The diagnosis and treatment of metastatic spinal tumor. Oncologist. 1999;4(6): 459-469.

23. Serlin RC, Mendoza TR, Nakamura Y, et al. When is cancer pain mild, moderate or severe? Grading pain severity by its interference with function. Pain. 1995;61(2):277-284.

24. Sawakami K, Yamazaki A, Ishikawa S, et al. Polymethylmethacrylate augmentation of pedicle screws increases the initial fixation in osteoporotic spine patients. J Spinal Disord Tech. 2012;25(2):E28-E35.

25. Versteeg AL, Verlaan JJ, de Baat P, et al. Complications after percutaneous pedicle screw fixation for the treatment of unstable spinal metastases. Ann Surg Oncol. 2016;23(7): 2343-2349.

26. Dea N, Versteeg A, Fisher C, et al. Adverse events in emergency oncological spine surgery: a prospective analysis. $J$ Neurosurg Spine. 2014;21(5):698-703.

27. Blinc A, Božič M, Vengust R, Stegnar M. Methyl-methacrylate bone cement surface does not promote platelet aggregation or plasma coagulation in vitro. Thromb Res. 2004;114(3): 179-184.

28. Krueger A, Bliemel C, Zettl R, Ruchholtz S. Management of pulmonary cement embolism after percutaneous vertebroplasty and kyphoplasty: a systematic review of the literature. Eur Spine J. 2009;18(9):1257-1265.

29. Wang LJ, Yang HL, Shi YX, et al. Pulmonary cement embolism associated with percutaneous vertebroplasty or kyphoplasty: a systematic review. Orthop Surg. 2012;4(3):182-189.

\section{Disclosures}

The authors report no conflict of interest concerning the materials or methods used in this study or the findings specified in this paper.

\section{Author Contributions}

Conception and design: Tatsui, Meleis, Larkin, Bastos, Muir. Acquisition of data: Meleis, Bastos, Muir. Analysis and interpretation of data: Meleis, Larkin, Bastos, Muir. Drafting the article: Meleis, Larkin, Bastos, Muir. Critically revising the article: Tatsui, Meleis, Larkin, Rao, Rhines, Cowles. Reviewed submitted version of manuscript: all authors. Approved the final version of the manuscript on behalf of all authors: Tatsui. Administrative/technical/ material support: Tatsui, Rao, Rhines, Cowles. Study supervision: Tatsui, Rao, Rhines, Cowles.

\section{Supplemental Information}

\section{Current Affiliations}

Dr. Rao: Department of Neurosurgery, Baylor College of Medicine, Houston, TX.

\section{Correspondence}

Claudio E. Tatsui: MD Anderson Cancer Center, Houston, TX. cetatsui@mdanderson.org. 